\title{
Melatonin Protects Human Adipose-Derived Stem Cells from Oxidative Stress and Cell Death
}

\author{
Shaun S. Tan, Xiaolian Han, Priyadharshini Sivakumaran, Shiang Y. Lim, Wayne A. Morrison \\ Bernard O'Brien Institute Department, St. Vincent's Institute of Medical Research, Melbourne, Australia
}

Background Adipose-derived stem cells (ASCs) have applications in regenerative medicine based on their therapeutic potential to repair and regenerate diseased and damaged tissue. They are commonly subject to oxidative stress during harvest and transplantation, which has detrimental effects on their subsequent viability. By functioning as an antioxidant against free radicals, melatonin may exert cytoprotective effects on ASCs.

Methods We cultured human ASCs in the presence of varying dosages of hydrogen peroxide and/or melatonin for a period of 3 hours. Cell viability and apoptosis were determined with propidium iodide and Hoechst 33342 staining under fluorescence microscopy.

Results Hydrogen peroxide (1-2.5 $\mathrm{mM}$ ) treatment resulted in an incremental increase in cell death. $2 \mathrm{mM}$ hydrogen peroxide was thereafter selected as the dose for co-treatment with melatonin. Melatonin alone had no adverse effects on ASCs. Co-treatment of ASCs with melatonin in the presence of hydrogen peroxide protected ASCs from cell death in a dose-dependent manner, and afforded maximal protection at $100 \mu \mathrm{M}(n=4$, one-way analysis of variance $\mathrm{P}<0.001$ ). Melatonin co-treated ASCs displayed significantly fewer apoptotic cells, as demonstrated by condensed and fragmented nuclei under fluorescence microscopy.

Conclusions Melatonin possesses cytoprotective properties against oxidative stress in human ASCs and might be a useful adjunct in fat grafting and cell-assisted lipotransfer.

Keywords Melatonin / Hydrogen peroxide / Mesenchymal stem cell / Cytoprotection
Correspondence: Shaun S. Tan Bernard O'Brien Institute Department, St. Vincent's Institute of Medical Research, 42 Fitzroy Street, VIC 3065 Tel: +613-9288-4018 Fax: +613-9416-0926 E-mail: shaunnietan@hotmail.com

No potential conflict of interest relevant to this article was reported.

\section{INTRODUCTION}

Adipose-derived stem cells (ASCs) have emerged as a key modality in tissue engineering and regenerative medicine. They have the versatility to develop into a variety of mature tissues and can be harnessed for therapeutic and reparative use in diseased or damaged tissues of the body. In addition, ASCs yield a comparatively greater proliferation capacity than bone marrowderived stem cells, whilst simultaneously providing a more convenient retrieval platform $[1,2]$. Nevertheless, a significant proportion of ASCs undergo cell death as a result of the hypoxic conditions and oxidative stress arising from the process of harvesting and/or transplantation [3]. In this study, we examined the role of melatonin, a human pineal gland hormone, in the protection of human ASCs against oxidative stress and cell death. Melatonin is of interest because of its purported anti-inflammatory and anti-apoptotic properties, which might allow it to act as a useful pharmacological adjunct for human ASCs [4]. In addition, it has been proven to be a safe, FDA-approved drug with few side effects and has since been incorporated into several human clinical trials [5]. We hypothesized that melatonin improves the efficacy of human ASCs by reducing oxidative stress and 
apoptosis, likely via antioxidant actions such as decreasing reactive oxidation species (ROS)-mediated injury to ASCs.

\section{METHODS}

\section{Isolation and culture of human ASCs}

Tissues were obtained with informed consent from three patients in accordance with the Australian National Health and Medical Research Council guidelines and approval from the St. Vincent's Health Human Research Ethics Committee. Patient 1 was a 27 -year-old male with adipose tissue obtained from the abdominal region. Patient 2 was a 52-year-old female with adipose tissue obtained from the abdominal and breast regions. $\mathrm{Pa}$ tient 3 was a 44-year-old female with adipose tissue obtained from the abdominal region. Isolation of primary ASCs was subsequently performed. Briefly, minced adipose tissues were digested with $0.1 \%$ type 1 collagenase (Worthington Biochemical) in phosphate-buffered saline (PBS) for 50 minutes. Upon centrifugation at $300 \mathrm{~g}$ for 5 minutes, cell pellets were resuspended in complete medium (Dulbecco's modified Eagle's medium [DMEM]-high glucose containing 10\% fetal calf serum and $1 \%$ antibiotic-antimycotic solution; Invitrogen), filtered through a $100 \mu \mathrm{m}$ nylon mesh, and later centrifuged at $700 \mathrm{~g}$ for $5 \mathrm{~min}$ utes. Cells were washed in PBS three times and the cell pellet was resuspended in complete DMEM and placed into tissue culture flasks for incubation overnight at $37^{\circ} \mathrm{C}$ in a humidified atmosphere containing $5 \% \mathrm{CO}_{2}$. The next day, fresh medium was applied and ASCs were harvested when they reached $90 \%$ confluence, based on their adherence to plastic. The cells were then frozen in liquid nitrogen. For our experiments, ASCs were thawed and cultured in complete DMEM. ASCs between passage 2 to 4 were used. Cells were trypsinized upon $80 \%$ confluence and seeded at a density of $1.5 \times 10^{4}$ in 96-well plates for all experiments the following day. Seeding was standardized to 5,000 cells per treatment group per well.

\section{Hydrogen peroxide and melatonin treatment}

Hydrogen peroxide was utilized as a model for oxidative stress and cell death $[6,7]$. ASCs were treated with varying doses (1 $\mathrm{mM}, 1.5 \mathrm{mM}, 2 \mathrm{mM}$, or $2.5 \mathrm{mM}$ ) of hydrogen peroxide and/or melatonin $(10 \mu \mathrm{M}, 50 \mu \mathrm{M}, 100 \mu \mathrm{M}$, or $200 \mu \mathrm{M})$ for a period of 3 hours. This time point was selected based on a previous study by Whittemore et al. [8] validating the effect of hydrogen peroxide on apoptosis and neuronal cell death.

\section{Assessment of ASC apoptosis}

Cells were stained with $10 \mu \mathrm{g} / \mathrm{mL}$ of Hoechst 33342 dye and counterstained with $5 \mu \mathrm{g} / \mathrm{mL}$ of propidium iodide (PI). Hoechst stain is a blue fluorescent dye that labels DNA in live or dead cells. In contrast, PI is a membrane impermeable dye that counterstains dead cells red. For each group, three random fields were captured using an Olympus IX71 microscope at 20 times magnification. The PI and Hoechst 33342 fields were merged and analyzed using the NIH Image J software [9]. The degree of apoptosis was calculated as the percentage of PI-positive cells over the total number of Hoechst 33342 cells. All experiments were performed at least thrice.

\section{Statistical analysis}

Data is expressed in terms of mean percentage, standard deviation and standard error of the mean. Statistical analyses were performed using analysis of variance (ANOVA) followed by Bonferroni's post-hoc test using the Statistical Package for the Social Sciences (IBM SPSS ver. 21.0; IBM Corp., Armonk, NY, USA). A P-value of $<0.05$ was considered statistically significant.

\section{RESULTS}

\section{Hydrogen peroxide induces oxidative stress and cell death}

1-2.5 $\mathrm{mM}$ of hydrogen peroxide treatment for 3 hours resulted in an incremental increase in cell death (control $2.48 \% \pm 0.96 \%$; $1 \mathrm{mM}$ hydrogen peroxide $8.92 \% \pm 5.59 \% ; 1.5 \mathrm{mM}$ hydrogen peroxide $48.13 \% \pm 9.65 \%$; $2 \mathrm{mM}$ hydrogen peroxide $78.14 \%$ $\pm 3.23 \%$; $2.5 \mathrm{mM}$ hydrogen peroxide $92.82 \% \pm 3.77 \%$; $\mathrm{n}=3$; one-way ANOVA between groups, $\mathrm{P}<0.001)$. Fig. 1 summarizes these results. Two $\mathrm{mM}$ hydrogen peroxide was thereafter selected as the dose for co-treatment with melatonin.

\section{Fig. 1. Effect of hydrogen peroxide on ASCs}

The effect of incremental concentrations of hydrogen peroxide on adipose-derived stem cells (ASCs) cell death. Incremental concentrations of hydrogen peroxide induced a greater degree of cell death in ASCs. Cells were stained with Hoechst 33342 and propidium iodide (PI), and the proportion of PI-stained cells was calculated. Error bars represent the standard error of the mean.

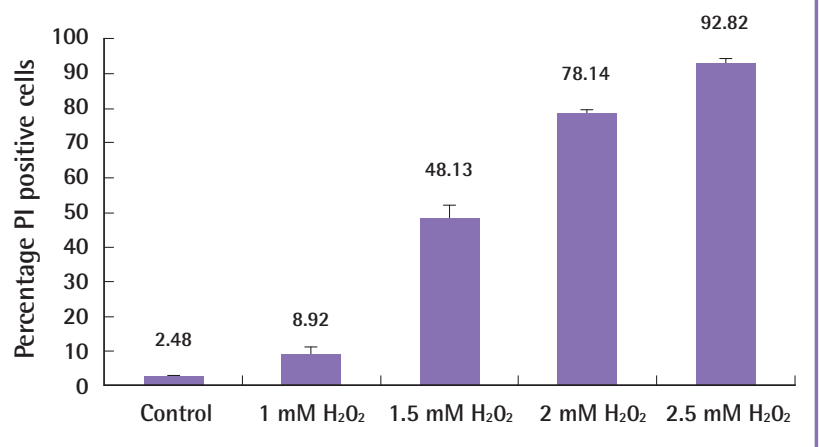




\section{Fig. 2. Melatonin has no toxic effect on ASCs}

Melatonin has no detrimental effect and does not induce cell death in human adipose-derived stem cells (ASCs). Varying concentrations of melatonin were cultured with human ASCs for a time period of 3 hours without hydrogen peroxide. There was no effect on cell death. The cells were stained with Hoechst 33342 and propidium iodide (PI) and the proportion of PI-stained cells was calculated. Error bars represent the standard error of the mean.

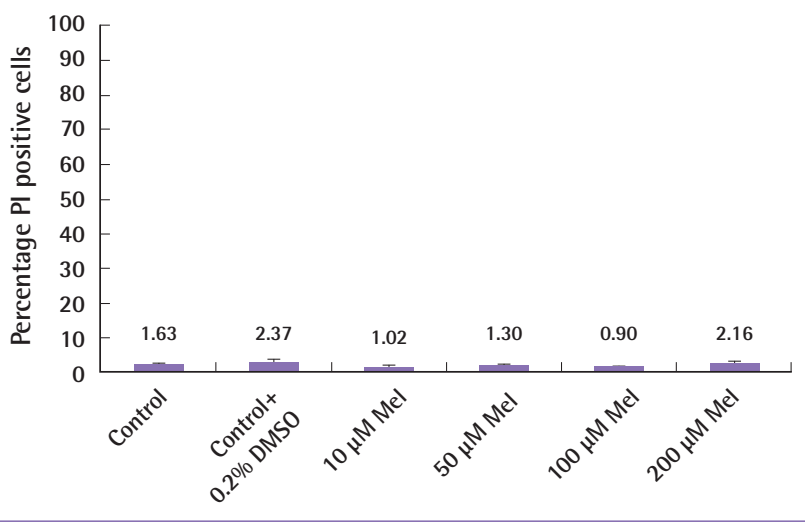

\section{Melatonin has no adverse effects on ASCs}

Different concentrations of melatonin $(10-200 \mu \mathrm{M})$ were cultured with ASCs in the absence of hydrogen peroxide for 3 hours. Melatonin and its vehicle, Dimethyl sulfoxide (DMSO), had no detrimental effects on ASCs, measured in terms of PI/Hoechst proportion and shown in Fig. 2 (control 1.63\% $\pm 1.51 \%$; control+0.2\% DMSO $2.37 \% \pm 1.72 \% ; 10 \mu \mathrm{M}$ melatonin $1.02 \% \pm$ $1.61 \%$; $50 \mu \mathrm{M}$ melatonin $1.3 \% \pm 1.46 \% ; 100 \mu \mathrm{M}$ melatonin $0.9 \%$ $\pm 1.16 \% ; 200 \mu \mathrm{M}$ melatonin $2.16 \% \pm 0.92 \% ; \mathrm{n}=4$, one-way ANOVA between groups, $\mathrm{P}=0.619$ ).

\section{Melatonin protects ASCs from hydrogen peroxide- induced cell death}

To determine the cytoprotective effect of melatonin against hydrogen peroxide-induced cell death, varying concentrations of melatonin were co-cultured with human ASCs in the presence of $2 \mathrm{mM}$ of hydrogen peroxide for a period of 3 hours. We demonstrate a dose-dependent cytoprotection of ASCs by melatonin against cell death induced by hydrogen peroxide (Fig. 3; proportion of PI/Hoechst cells in control 1.63\% $\pm 1.51 \%$; 2 $\mathrm{mM}$ hydrogen peroxide+0.2\% DMSO $77.11 \% \pm 3.48 \% ; 2 \mathrm{mM}$ hydrogen peroxide $10 \mu \mathrm{M}$ melatonin $74.39 \% \pm 2.45 \%$; $2 \mathrm{mM}$ hydrogen peroxide $+50 \mu \mathrm{M}$ melatonin $59.43 \% \pm 5.26 \% ; 2 \mathrm{mM}$ hydrogen peroxide $+100 \mu \mathrm{M}$ melatonin $43.58 \% \pm 1.82 \% ; 2 \mathrm{mM}$ hydrogen peroxide $+200 \mu \mathrm{M}$ melatonin $57.12 \% \pm 5.35 \% ; \mathrm{n}=4$, one-way ANOVA P $<0.001$ ).

Significantly, $100 \mu \mathrm{M}$ of melatonin induced the most potent cytoprotection for ASCs against hydrogen peroxide-induced cell death, compared to hydrogen peroxide alone (Bonferonni
Fig. 3. Dose-dependent cytoprotective effects of Melatonin

Melatonin protects human adipose-derived stem cells (ASCs) against cell death induced by hydrogen peroxide in a dose-dependent manner. Melatonin has a dose-dependent effect of cytoprotection for ASCs against hydrogen peroxide, as shown with increasing concentrations of melatonin $(10-200 \mu \mathrm{M})$. The melatonin dose with the strongest protection was $100 \mu \mathrm{M}$. Error bars represent the standard error of the mean. $\mathrm{Pl}$, propidium iodide.

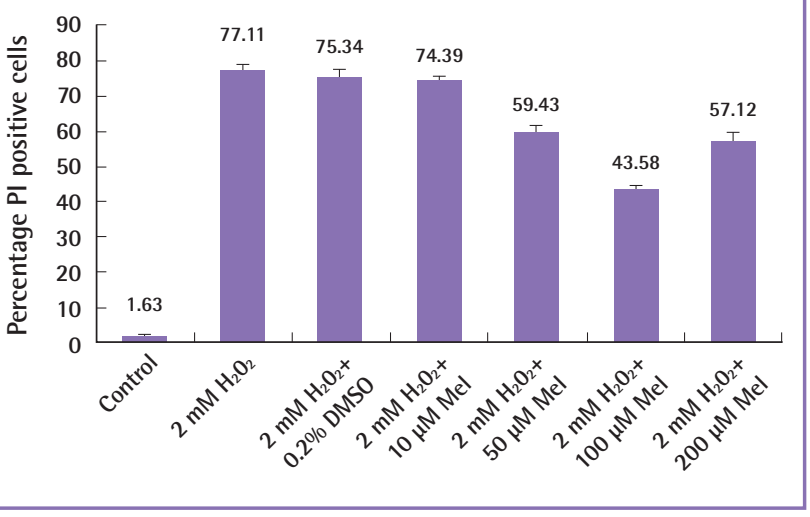

post-hoc test $\mathrm{P}<0.001)$. In hydrogen peroxide-treated ASCs, a considerable proportion of cells displayed condensed and fragmented nuclei, consistent with characteristics of apoptosis. The addition of melatonin significantly reduced the proportion of apoptotic cells. Fig. 3 charts the dose-dependent response of melatonin and Fig. 4 shows the fluorescence microscopy images taken at 20 times magnification. Fig. $4 \mathrm{~A}-\mathrm{C}$ demonstrates that human ASCs that did not receive any hydrogen peroxide treatment did not undergo cell death. Conversely, the addition of hydrogen peroxide to both control (Fig. 4D) and vehicle groups (Fig. 4E) induced apoptosis whilst the group of human ASCs exposed to $100 \mu \mathrm{M}$ of melatonin (Fig. 4F) was rescued from cell death.

\section{DISCUSSION}

ASCs are of considerable interest to the plastic surgery community and have been successfully incorporated into fat grafting. Fat grafting is a key technique in soft tissue reconstruction using nonvascularized adipose tissue. It has a multitude of applications to conditions such as burns and irradiation wounds, trauma, postcancer resection and ageing [10]. Recently, Yoshimura and colleagues demonstrated that autologous fat grafting with lipoaspirate enriched with stromal vascular fraction, a rich source of ASCs, improves clinical outcomes in breast augmentation and facial lipoatrophy patients $[11,12]$. This has been termed "cellassisted lipoaspirate" and proponents believe this may augment the eventual volume and long-term stability of fat grafts.

Success, however, is often limited by poor survival of the ASCs 


\section{Fig. 4. PI and Hoechst stains of ASCs}

Fluorescence microscopy of Hoechst and propidium iodide (PI)-stained, merged images of human adipose stem cells taken at 20 times magnification. Human adipose-derived stem cells (ASCS) that did not receive hydrogen peroxide treatment did not undergo cell death (A-C). Hydrogen peroxide induced apoptosis in both control (D) and vehicle groups (E), while $100 \mu \mathrm{M}$ melatonin (E) rescued a significant proportion of human ASCs from cell death. (A) Control human ASCs: no treatment. (B) 0.2\% Dimethyl sulfoxide (DMSO) (vehicle) group of human ASCs: no hydrogen peroxide treatment. (C) $100 \mu \mathrm{m}$ melatonin group of human ASCs: no hydrogen peroxide treatment. (D) Human ASCs treated with 2 mM hydrogen peroxide for 3 hours. (E) Human ASCs treated with 0.2\% DMSO+2 mM hydrogen peroxide for 3 hours. (F) Human ASCs treated with $100 \mu$ M melatonin+2 $\mathrm{mM}$ hydrogen peroxide for 3 hours.
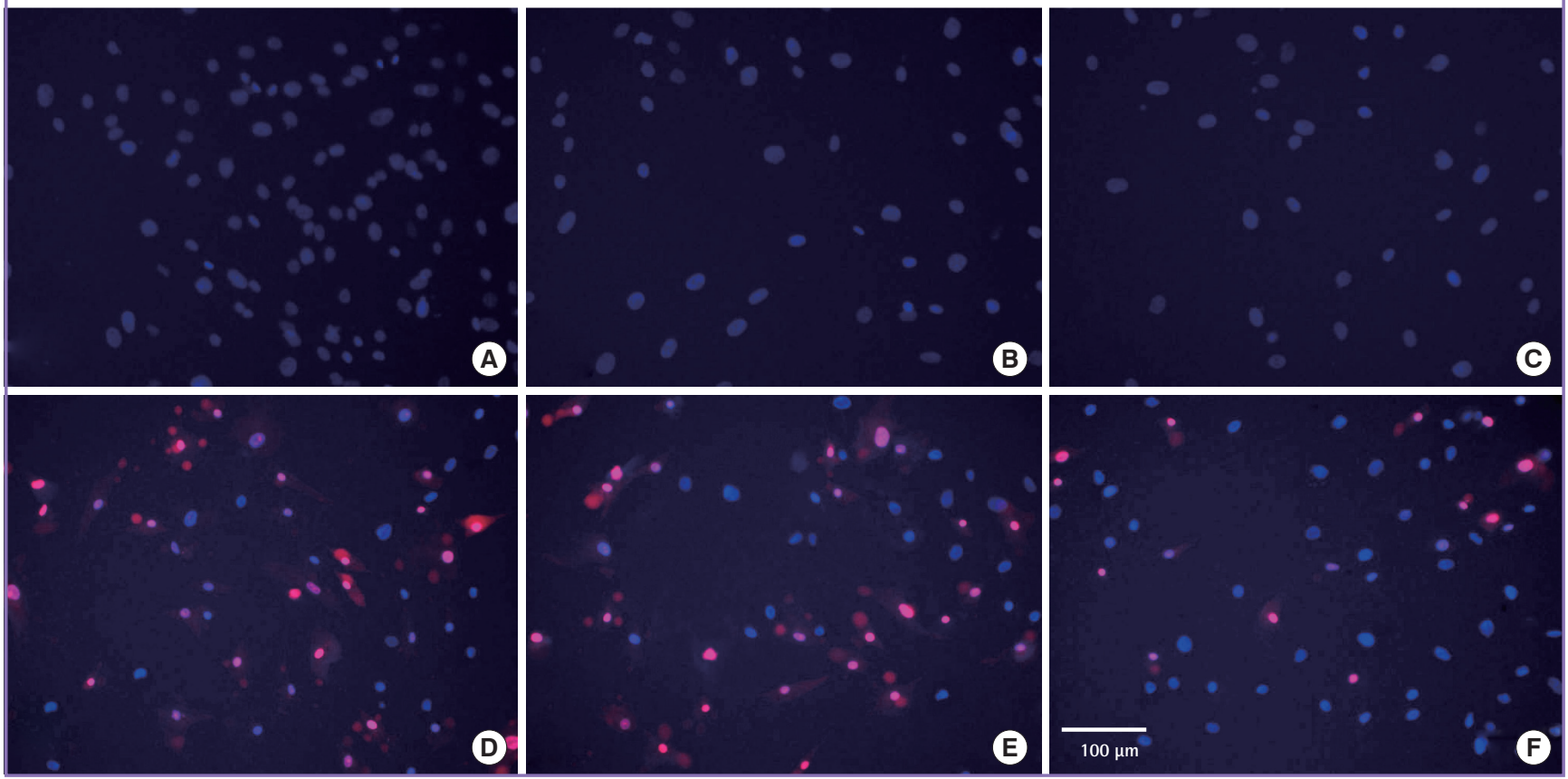

and unpredictability of the fat graft. A significant proportion of the engrafted fat and ASCs undergoes necrosis due to various factors such as trauma, ischemia, oxidative stress and inflammation [10]. To this end, novel strategies have been employed to enhance the cytoprotection of stem cells at the target site, which include ischemic preconditioning, pharmacological therapy, refinement of liposuction techniques to minimize trauma, as well as the addition of growth factors $[10,13,14]$.

First discovered in 1958, melatonin has become a useful sleep aid due to its ability to promote normal circadian rhythm in the human pineal gland. Importantly, melatonin is an FDA-approved drug synthesized as a natural hormone, and has purported antiapoptotic, anti-inflammatory, and antioxidant properties $[14,15]$. In particular, it has minimal adverse effects on the human body, including non-specific symptoms such as nausea, headaches and dizziness [4]. As such, it has a high safety profile and has been included in many human clinical trials. Luchetti and colleagues propose that the anti-apoptotic effects of melatonin act by limiting the production of reactive oxidation species, thus protecting mitochondrial membranes [4]. In addition, it upregulates the transcriptional and post-translational glutathione system, which is essential for defense, repair and anti-apoptosis.
Recent studies have demonstrated the efficacy of melatonin in improving the therapeutic potency of ASCs in myocardial infarction and acute lung injury within rat models $[16,17]$.

Hydrogen peroxide was chosen as a model of oxidative stress in our study because it induces generation of ROS, leading to intracellular DNA oxidation, lipid peroxidation and ultimately cell death [6]. This simulates the harsh, pro-oxidant environment ASCs are often subject to in various clinical scenarios such as fat grafting, or animal experiments such as ASC injections for myocardial infarction [18]. Admittedly, factors apart from oxidative stress, such as ischemia and trauma, may contribute to ASC death and these will be useful to investigate in further experiments. The rationale for co-treating ASCs with melatonin at the same time as hydrogen peroxide is that it closely mimics the clinical scenario of fat grafting. It would be impractical for a surgeon to pre-treat a fat graft with a drug for a considerable amount of time before re-injection into a patient who is currently anesthetized.

The results of our study firstly characterize the effect of incremental concentrations of hydrogen peroxide, an agent of oxidative stress, on ASC cell death. Secondly, our results are novel in showing that melatonin provides a dose-dependent rescue of 
ASCs from cell death induced by oxidative stress. In particular, it is of interest that $100 \mu \mathrm{M}$ of melatonin confers greater cytoprotection on ASCs as compared to $200 \mu \mathrm{M}$ of melatonin. The effect of melatonin represents a bell-shaped dose-response relationship [19], such that the cytoprotective effect of the drug on ASCs is maximal at $100 \mu \mathrm{M}$ before declining at higher doses. This is because melatonin has complex biological effects and multiple binding sites (melatonin receptors 1, 2, and 3) and may lose activity at higher concentrations [20]. Finally, we determined that melatonin has no toxic effect on human ASCs and as such has a good safety profile. Taken together, the findings of this study provide support to the use of melatonin-treated ASCs and fat grafts in an in vivo model as well as a human clinical trial.

\section{REFERENCES}

1. Fraser JK, Wulur I, Alfonso Z, et al. Fat tissue: an underappreciated source of stem cells for biotechnology. Trends Biotechnol 2006;24:150-4.

2. Li CY, Wu XY, Tong JB, et al. Comparative analysis of human mesenchymal stem cells from bone marrow and adipose tissue under xeno-free conditions for cell therapy. Stem Cell Res Ther 2015;6:55.

3. Monsel A, Zhu YG, Gennai S, et al. Cell-based therapy for acute organ injury: preclinical evidence and ongoing clinical trials using mesenchymal stem cells. Anesthesiology 2014; 121:1099-121.

4. Luchetti F, Canonico B, Betti M, et al. Melatonin signaling and cell protection function. FASEB J 2010;24:3603-24.

5. Sanchez-Barcelo EJ, Mediavilla MD, Tan DX, et al. Clinical uses of melatonin: evaluation of human trials. Curr Med Chem 2010;17:2070-95.

6. Deavall DG, Martin EA, Horner JM, et al. Drug-induced oxidative stress and toxicity. J Toxicol 2012;2012:645460.

7. Yeh CH, Ma KH, Liu PS, et al. Baicalein Decreases Hydrogen Peroxide-Induced Damage to NG108-15 Cells via Upregulation of Nrf2.J Cell Physiol 2015;230:1840-51.

8. Whittemore ER, Loo DT, Watt JA, et al. A detailed analysis of hydrogen peroxide-induced cell death in primary neuronal culture. Neuroscience 1995;67:921-32.
9. Schneider CA, Rasband WS, Eliceiri KW. NIH Image to ImageJ: 25 years of image analysis. Nat Meth 2012;9:671-5.

10. Mashiko T, Yoshimura K. How does fat survive and remodel after grafting? Clin Plast Surg 2015;42:181-90.

11. Yoshimura K, Sato K, Aoi N, et al. Cell-assisted lipotransfer for facial lipoatrophy: efficacy of clinical use of adipose-derived stem cells. Dermatol Surg 2008;34:1178-85.

12. Yoshimura K, Sato K, Aoi N, et al. Cell-assisted lipotransfer for cosmetic breast augmentation: supportive use of adiposederived stem/stromal cells. Aesthetic Plast Surg 2008;32:4855.

13. Hsiao ST, Dilley RJ, Dusting GJ, et al. Ischemic preconditioning for cell-based therapy and tissue engineering. Pharmacol Ther 2014;142:141-53.

14. Lim HD, Kim YS, Ko SH, et al. Cytoprotective and anti-inflammatory effects of melatonin in hydrogen peroxide-stimulated CHON-001 human chondrocyte cell line and rabbit model of osteoarthritis via the SIRT1 pathway. J Pineal Res 2012;53:225-37.

15. Kim SH, Lee SM. Cytoprotective effects of melatonin against necrosis and apoptosis induced by ischemia/reperfusion injury in rat liver. J Pineal Res 2008;44:165-71.

16. Chen HH, Chang CL, Lin KC, et al. Melatonin augments apoptotic adipose-derived mesenchymal stem cell treatment against sepsis-induced acute lung injury. Am J Transl Res 2014;6:439-58.

17. Zhu P, Liu J, Shi J, et al. Melatonin protects ADSCs from ROS and enhances their therapeutic potency in a rat model of myocardial infarction. J Cell Mol Med 2015;19:2232-43.

18. Amiri F, Jahanian-Najafabadi A, Roudkenar MH. In vitro augmentation of mesenchymal stem cells viability in stressful microenvironments: in vitro augmentation of mesenchymal stem cells viability. Cell Stress Chaperones 2015;20: 237-51.

19. Reynolds AR. Potential relevance of bell-shaped and u-shaped dose-responses for the therapeutic targeting of angiogenesis in cancer. Dose Response 2009;8:253-84.

20. Owen SC, Doak AK, Ganesh AN, et al. Colloidal drug formulations can explain "bell-shaped" concentration-response curves. ACS Chem Biol 2014;9:777-84. 